\title{
Psychological debriefing in schools
}

\author{
Magdalena Szumilas MSc, Yifeng Wei MEd, Stan Kutcher MD
}

Previously published at www.cmaj.ca

$\mathrm{S}$ chool staff, administrators and health care providers are often called upon to deal with suicides, violent incidents and emergencies affecting students. Critical incident stress debriefing and management are techniques of psychological debriefing commonly applied following traumatic events (Box 1). ${ }^{1,2}$ The widespread acceptance of these techniques was based on the assumption that they were effective and safe and would substantially reduce acute symptoms of distress associated with exposure to traumatic events, thereby decreasing the risk of post-traumatic stress disorder. However, recent research suggests that these interventions are ineffective and may be harmful. ${ }^{1,3-7}$

Cochrane reviews of single-session and multiple-session interventions for psychological debriefing indicated that they neither prevent post-traumatic stress disorder nor significantly reduce acute psychological distress when compared with control treatments in adults. ${ }^{1.3}$ Further, the reviews recommended against routine use of psychological debriefing to prevent post-traumatic stress disorder. Other analyses showed that people who received psychological debriefing exhibited more severe symptoms of post-traumatic stress disorder than controls; ${ }^{7}$ that the intervention increased the risk of the stress disorder; ${ }^{5}$ and that critical incident stress debriefing, in particular, was potentially harmful. ${ }^{4}$ These findings are in stark contrast to the positive results noted in earlier studies by the creators and marketers of critical incident stress debriefing and management. ${ }^{2,8}$

Research on the effectiveness and safety of such interventions in schools is very limited. ${ }^{9}$ Authors in one Cochrane review noted that they were "unaware of the evidence base surrounding debriefing in children" (16 years or

\section{Box 1: Psychotherapeutic techniques}

Psychological debriefing: Single-session individual psychological intervention that involves reworking, reliving or recollection of the trauma and subsequent emotional reactions.

Critical incident stress debriefing: Used in the 1980s to describe a small-group-based multicomponent program for crisis intervention designed for emergency service workers (secondary trauma victims). ${ }^{2}$

Critical incident stress management: Introduced in the 1990 s to refer to the "overarching umbrella program/system" as well as group-based psychological debriefing to remediate the impact of traumatic incidents.

\section{Key points}

- Critical incident stress debriefing and management are interventions for psychological debriefing often used in schools for students affected by suicide, accidental death and trauma.

- There is a lack of controlled studies that prove the effectiveness or safety of these interventions in schools.

- Given evidence that these interventions are ineffective and potentially harmful in adults, there is no compelling reason to implement them in schools.

- Psychological first aid and cognitive behavioural intervention for trauma in schools are showing promising results.

younger). ${ }^{1}$ Despite evidence of ineffective and indeed harmful effects in adults and the absence of evidence in children, some schools use these interventions following suicide, accidental death or other traumatic events among their students. Several provinces recommend these interventions in school policy documents, and some school boards employ crisis or grief counsellors. In this light, perhaps we should consider McNally and colleagues' comments about companies and employees: "Given the absence of data showing that debriefing works, and given some studies suggesting that debriefing may impede natural recovery from trauma, companies may be at heightened risk [for liability] if they do debrief their employees, especially if they fail to provide informed consent." 6

The evidence clearly points to the ineffectiveness of these interventions in preventing post-traumatic stress disorder or any other psychiatric disorder in adults. Further, with the lack of controlled studies in schools, it is not possible to endorse the use of psychological debriefing in schools on scientific, ethical or legal grounds.

So what should mental health professionals and policymakers consider as appropriate crisis intervention in schools? Given our current knowledge, it is prudent to develop interventions that promote the following empiri-

From the Sun Life Financial Chair in Adolescent Mental Health Team, Dalhousie University and IWK Health Centre (Szumilas, Wei, Kutcher); and the Department of Psychiatry and the WHO Collaborating Centre for Mental Health Training and Policy (Kutcher), Dalhousie University, Halifax, NS

CMAJ 2010. DOI:10.1503/cmaj.091621

All editorial matter in CMAJ represents the opinions of the authors and not necessarily those of the Canadian Medical Association. 
cally supported principles: a sense of safety; calmness; a sense of self and community efficacy; connectedness; and hope.$^{10}$ Preliminary analyses of two programs developed according to these principles show promise of effectiveness: Psychological First Aid ${ }^{11}$ could be applied immediately after an incident, and Cognitive Behavioural Intervention for Trauma in Schools ${ }^{12}$ could be provided to students who experience psychological distress weeks after a trauma has passed. These interventions could also form part of screening strategies or training of school personnel to help them identify students most at risk.

Mental health interventions should be based on best scientific evidence. Our analysis shows no evidence to support the use of psychological debriefing in schools. There is an urgent need to conduct methodologically sound evaluations of psychological debriefing and other mental health interventions in schools before they are widely implemented and to make the results easily available to both educators and health professionals.

Competing interests: None declared.

Contributors: Magdalena Szumilas and Yifeng Wei conducted the literature review and analysis and wrote the manuscript. Stan Kutcher conceptualized the commentary and made substantial contributions and revisions to the manuscript. All of the authors approved the final version submitted for publication.

Acknowledgement: The authors thank Alan McLuckie for his help with the organization of the manuscript.

\section{REFERENCES}

1. Rose S, Bisson J, Churchill R, et al. Psychological debriefing for preventing post traumatic stress disorder (PTSD) [review]. Cochrane Database Syst Rev 2002;(2): CD000560.

2. Everly GS Jr, Flannery RB Jr, Eyler VA. Critical incident stress management (CISM): a statistical review of the literature. Psychiatr Q 2002;73:171-82.

3. Roberts NP, Kitchiner NJ, Kenardy J, et al. Multiple session early psychological interventions for the prevention of post-traumatic stress disorder [review]. Cochrane Database Syst Rev 2009;(3):CD006869.

4. Lilienfeld SO. Psychological treatments that cause harm. Perspect Psychol Sci 2007;2:53-70.

5. Cuijpers P, Van Straten A, Smit F. Preventing the incidence of new cases of mental disorders: a meta-analytic review. J Nerv Ment Dis 2005;193:119-25.

6. McNally RJ, Bryant RA, Ehlers A. Does early psychological intervention promote recovery from posttraumatic stress? Psychol Sci Public Interest 2003;4:45-79.

7. Litz BT, Gray MJ, Bryant RA, et al. Early intervention for trauma: current status and future directions. Clin Psychol Sci Pract 2002;9:112-34.

8. Everly GS Jr, Boyle SH. Critical incident stress debriefing (CISD): a meta-analysis. Int J Emerg Ment Health 1999;1:165-8.

9. Wethington HR, Hahn RA, Fuqua-Whitley DS, et al. The effectiveness of interventions to reduce psychological harm from traumatic events among children and adolescents: a systematic review. Am J Prev Med 2008;35:287-313.

10. Hobfoll SE, Watson P, Bell CC, et al. Five essential elements of immediate and midterm mass trauma intervention: empirical evidence. Psychiatry 2007;70:283-315

11. Ruzek JI, Brymer MJ, Jacobes AK, et al. Psychological first aid. J Ment Health Counsel 2007;29:17-49.

12. Ngo V, Langley A, Kataoka SH, et al. Providing evidence-based practice to ethnically diverse youths: examples from the Cognitive Behavioral Intervention for Trauma in Schools (CBITS) program. J Am Acad Child Adolesc Psychiatry 2008;47:858-62.

Correspondence to: Ms. Magdelena Szumilas, Sun Life Financial Chair in Adolescent Mental Health, Dalhousie University and IWK Health Centre, 5850 University Ave., PO Box 9700, Halifax NS B3K6R8; magdaszumilas@gmail.com
Cervarix is is a prophylactic vaccine. It does not prevent progression of HPV-related lesions present at vaccination. Cervarix does not protect against all oncogenic HPV types and may not prevent infection with HPV I 6/I 8 or subsequent progression to cervical carcinoma in all vaccinees. Cervarix is not a treatment for current HPV infection, precancerous lesions, or cervical cancer. Vaccination is for primary prevention and is not a substitute for regular cervical screening (secondary prevention) or for precautions against exposure to HPV and sexually transmitted diseases.

Vaccination should not be undertaken in pregnant women and vaccinees should be advised to take adequate precautions to avoid pregnancy for 2 months following vaccination.

The most commonly reported adverse events within 7 days of vaccination with Cervarix ${ }^{\text {TM }} /$ control were: Local [pain (91.8\%/87.2\%), redness (48.0\%/24.4\%) and swelling (44.1\%/21.3\%)]; General [fatigue (55.0\%/53.6\%), headache (53.4\%/6l.4\%)].*

Please see the full Product Monograph.

${ }^{*}$ Control $=\mathrm{Al}(\mathrm{OH})_{3}$ control containing $500 \mu \mathrm{g} \mathrm{Al}(\mathrm{OH})_{3}$ Reference: I. Data on file. GSKBio_WWMA DoF025_5_2010.

Cervarix ${ }^{\mathrm{Th}}$ is used under license by GlaxoSmithKline Inc. (C)20 I0 GlaxoSmithKline Inc. All rights reserved.

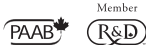

